Article

\title{
Optimization of Ultrasonic Extraction to Obtain Erinacine A and Polyphenols with Antioxidant Activity from the Fungal Biomass of Hericium erinaceus
}

\author{
Mihai-Vlad Valu ${ }^{1,2}{ }^{\mathbb{D}}$, Liliana Cristina Soare ${ }^{1}$, Nicoleta Anca Sutan ${ }^{1}$, Catalin Ducu ${ }^{2}$, \\ Sorin Moga ${ }^{2}$, Lucian Hritcu ${ }^{3}{ }^{-}$, Razvan Stefan Boiangiu ${ }^{3}(-)$ and Simone Carradori ${ }^{4, *}$ \\ 1 Department of Natural Sciences, Faculty of Science, University of Pitesti, Targu din Vale Street, \\ 110040 Pitesti, Romania; mihai.valu@upit.ro (M.-V.V.); cristina.soare@upit.ro (L.C.S.); \\ anca.sutan@upit.ro (N.A.S.) \\ 2 Regional Research and Development Center for Innovative Materials, \\ University of Pitesti, Products, and Processes from Automotive Industry, 11 Doaga Street, Arges, \\ 110440 Pitesti, Romania; catalin.ducu@upit.ro (C.D.); sorin.moga@upit.ro (S.M.) \\ 3 Department of Biology, Alexandru Ioan Cuza University of Iasi, Bd. Carol I, No. 11, 700506 Iasi, Romania; \\ hritcu@uaic.ro (L.H.); razvan.boiangiu@student.uaic.ro (R.S.B.) \\ 4 Department of Pharmacy, “G. d'Annunzio" University of Chieti-Pescara, Via dei Vestini 31, 66100 Chieti, Italy \\ * Correspondence: simone.carradori@unich.it
}

Received: 18 November 2020; Accepted: 15 December 2020; Published: 18 December 2020

\begin{abstract}
Hericium erinaceus is a medicinal fungal species that produces the active biological metabolite erinacine A with strong antioxidant activity. The classical extraction techniques used to date to obtain metabolites from this fungal species require high consumption of resources and energy and, in the end, prove to be expensive and inefficient, especially on a biomedical scale. The aim of this research is based on the development of an ultrasonic extraction (UE) method for the identification and extraction of biological compounds with high antioxidant activity from the mycelia of $H$. erinaceus biomass developed through a solid cultivation process. The extraction process was optimized by varying parameters to determine the best extraction yield of metabolites involved in such antioxidant activity, using the response surface methodology (RSM). The physicochemical analyses were oriented towards the investigation of polyphenols, flavonoids, and the diterpenoid erinacine A. It is highlighted that there is a very good mutual connection between the concentration of polyphenols and flavonoids in the extracts studied and the diterpenoid erinacine A. Also, this study describes an efficient and qualitative extraction method for extracting natural antioxidants from the H. erinaceus mushroom, since toxic solvents were not used in the developed extraction procedure. This biomass can be used both as a food source and as a possible phytotherapeutic tool in the prevention or treatment of various neurodegenerative disorders that require drugs with strong antioxidant activity.
\end{abstract}

Keywords: Hericium erinaceus; ultrasonic extraction; erinacine A; HPLC analysis; antioxidant activity

\section{Introduction}

Certain edible mushrooms can be classified as medicinal, having many biologically active compounds for human health, due to their consistency [1]. Before the advancement of modern medicine, people relied on natural remedies with antioxidant activity for the prevention and/or treatment of various diseases, including Parkinson's disease, Alzheimer's disease (AD), cancer, etc. [2]. The therapeutic potential of secondary metabolites in mushroom species has received special attention 
recently, especially regarding neurodegenerative diseases such as Alzheimer's disease [3,4]. For example, in the case of $\mathrm{AD}$, no safe means of prevention is known, and it is a progressive neurodegenerative disorder with etiopathogenesis involving the participation of numerous risk factors [5]. It is important to point out that extraction is considered the first step in advancing the development of the main traditional mushroom-based remedies. Considerable efforts have been made by researchers to find the most effective extraction methods having better efficiency and providing extracts endowed with high bioactivity [6,7]. It should be noted that attempts are still being made to develop an extraction method that is suitable for mushrooms, applying different parameters for high efficiency [8]. Many published studies have reported that ultrasonic extraction can improve the extraction rate of active biological heat-sensitive ingredients under low-temperature processing conditions, making it more effective than conventional extraction techniques [9]. The mechanical effect of ultrasonication causes the penetration of solvents into tissue cells, improved mass transfer, and tearing of the cell wall, which is favorable to the release of tissue cell content and can reduce the processing time and required amount of solvents compared to traditional extraction methods [10]. Hericium is a genus of edible mushrooms of the Hericiaceae family. The genus Hericium produces phytochemical substances, erinacines and hericenones, with antioxidant activity, but also various polysaccharides [11]. Erinacine A (Figure 1) was isolated from the fungal species Hericium erinaceus; its chemical structure is deposited in PubChem with the indication $9,867,477$.

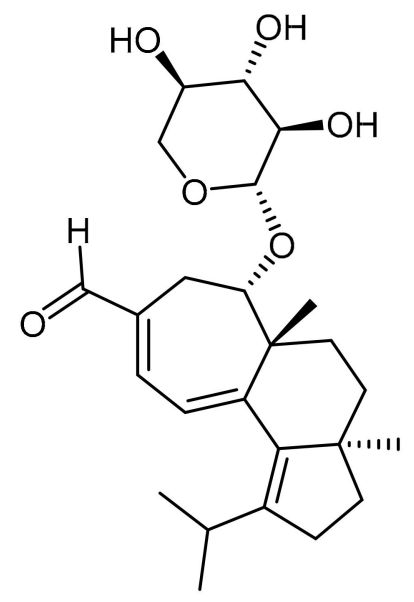

Figure 1. Chemical structure of erinacine A. Chemical Formula: $\mathrm{C}_{25} \mathrm{H}_{36} \mathrm{O}_{6}(432.5 \mathrm{~g} / \mathrm{mol}$.).

Mainly, the antioxidant activity of the H. erinaceus species is due to the presence of polysaccharides ( $\beta$-glucans) and diterpenoids (hericenons, erinacines), but several authors have stated that there is a close link between the antioxidant activity of $H$. erinaceus and the presence of flavonoids and polyphenols [12,13]. It has been noted that the biosynthesis of erinacine $\mathrm{A}$ in submerged culture can lower production costs because the chemical synthesis of diterpenoids is a long, multistage process and provides poor yield $[13,14]$. Most scientific studies on the antioxidant properties of $H$. erinaceus have described classical extraction techniques, although in the literature there are different modern methods of extraction. Therefore, an additional task of this study was to investigate the antioxidant properties of H. erinaceus extracts obtained by ultrasonic extraction. At the same time, given that study results have been contradictory concerning the presence of flavonoids and polyphenols with antioxidant activity in mushroom species $[15,16]$, this issue has aroused our interest to investigate various physicochemical methods for the possible presence of these biocompounds. Besides this, the number of components with antioxidant effects was determined. It has been shown that the antioxidant effects of several mushrooms are also related to their total phenolic and flavonoid contents [17]. Further, it is expected that the antioxidant activity is due to the high yield of extracted erinacine A as identified by HPLC. The extraction parameters (extraction time, ethanol concentration, and solvent/material ratio) were also investigated to maximize the extraction yield in terms of the number of metabolites and to establish 
optimal extraction conditions. The response surface method (RSM) is an innovative statistical method used to optimize processing parameters. Therefore, RSM is used to speed up and optimize operating processes to reduce the required time, energy, and raw materials. The response surface methodology was applied to optimize the ultrasonic extraction parameters to obtain high yield and content of antioxidant compounds of HE. The main advantage of RSM is that it can reduce the required number of experiments and highlight the relationship between response and variables. This is the first report on the determination of erinacine $\mathrm{A}$, as well as on the optimization of the ultrasonic extraction conditions of metabolites with antioxidant activity. In this work, we aimed to enhance the phenolic, flavonoid, and erinacine A contents and antioxidant activity of H. erinaceus biomass under ultrasonic extraction.

\section{Materials and Methods}

\subsection{Chemicals and Reagents}

Erinacine A, not commercially available, was obtained from the fungal biomass of $H$. erinaceus as previously reported [18]. Casein peptone, glucose, sodium chloride $(\mathrm{NaCl})$, monopotassium phosphate $\left(\mathrm{KH}_{2} \mathrm{PO}_{4}\right)$, hydrochloric acid $(\mathrm{HCl})$, and nitric acid $\left(\mathrm{HNO}_{3}\right)$ were obtained from ThermoFisher Scientific (Darmstadt, Germany). Folin-Ciocalteu reagent, 2,2-diphenyl-1-picrylhydrazyl (DPPH), quercetin, gallic acid, L-ascorbic acid, galantamine, acetylthiocholine iodide, butyrylthiocholine chloride, trichloroacetic acid (TCA), and iron(III) chloride $\left(\mathrm{FeCl}_{3}\right)$ were all purchased from Sigma-Aldrich (Steinheim, Germany), and commercial ethyl alcohol (96\%) was purchased from Prodvinalco S.A. Romania. The other substances were of analytical quality.

\subsection{Solid-State Cultivation of H. erinaceus Biomass}

In this research, the H. erinaceus biomass was developed on a solid culture medium under controlled conditions to obtain the diterpenoid erinacine A, and the antioxidant activity was investigated. The biomass was developed in the laboratory University of Agronomic Sciences and Veterinary Medicine of Bucharest (USAMV, Bucharest) for 21 days following an adapted protocol [19]. In a 9.0 L glass vessel, the culture medium was prepared from casein peptone $12.16 \mathrm{~g} / \mathrm{L}$, glucose $66.88 \mathrm{~g} / \mathrm{L}$, $\mathrm{NaCl} 1.46 \mathrm{~g} / \mathrm{L}$, and $\mathrm{KH}_{2} \mathrm{PO}_{4} 1.0 \mathrm{~g} / \mathrm{L}$ with a pH of 4.5 [19]. Moreover, to enrich the yield of the compounds of interest, namely, erinacine A, flavonoids, and polyphenols, contents of $0.5 \%$ yeast extract, $4 \%$ glucose, $0.5 \%$ soy powder, and $1 \%$ oats were added to the culture medium.

\subsection{Ultrasonic Extraction Procedure}

The technology for obtaining and concentrating the bioactive products of the H. erinaceus biomass was developed based on the principles of the disintegration of cell structures using ultrasound waves. The device used for extraction was a Hielscher ultrasonic processor (Hielscher UIP1000hdT Berlin, Germany), with a sonotrode of $40 \mathrm{~mm}$ diameter, 1000 Watts, $20 \mathrm{kHz}$, and adjustable amplitude (amplitude ratio 1:0.7). Before the extraction experiments, the ultrasonic processor was calibrated to find the power consumption of the equipment. During the sonication process, this value was automatically deducted from the gross energy consumption, thus allowing the net power delivered to the extraction medium to be found. During the experiments, the samples were placed in an ice bag with continuous magnetic stirring to maintain a low sample temperature. After completion of the extraction, the samples were vacuum filtered and then centrifuged $(2500 \times \mathrm{g}$ for $5 \mathrm{~min})$. A rotary evaporator (Heidolph Hei-VAP Core, Schwabach, Germany) was used for water and alcohol elimination from the supernatants. The remaining water and alcohol residues from the samples were subjected to the lyophilization process.

\subsection{Lyophilization of Samples}

The last stage consisted of lyophilization of the samples (Christ Alpha 1-2 LDplus, Osterode, Germany) to obtain a powder (permanently prepared, shredded as particles) of the fungal material. 
The aim of the lyophilization was to obtain high-quality extracts to maintain extraction efficiency, with a higher content in active principles due to freeze-drying [20]. The working procedure was optimized and consisted of subjecting the liquid samples in the Petri dishes $(7 \mathrm{~mm})$ to freezing at the vaporization temperature of $-70{ }^{\circ} \mathrm{C}$ for $2 \mathrm{~h}$ and freeze-off at $-55^{\circ} \mathrm{C}$ under vacuum for $48 \mathrm{~h}$. Vacuum in the enclosure was achieved at a constant pressure of $400 \mu$ Bar. Finally, the material was restored to room temperature, resulting in a powder extract. An important aspect of freeze-making is that it limits oxidative changes in metabolites since the oxygen concentration is very low under vacuum.

\subsection{Optimization of the Extraction Procedure Using the RSM Model}

Response surface methodology (RSM) was applied to investigate the impact of three independent variables on the total phenolics and yield of $H$. erinaceus extract. The factors that influence the extraction quality are solvent concentration $(\%, \mathrm{X} 1)$, extraction time ( $\mathrm{min}, \mathrm{X} 2)$, and the solvent-material ratio $(\mathrm{mL} / \mathrm{g}, \mathrm{X} 3)$. The temperature was not taken into account in this experimental model because the extraction time started once the desired temperature was reached. The experiments were performed using the Box-Behnken Design (BBD). Three variables were taken into consideration to optimize the best combination of extraction parameters for extract yield and total phenolic content. The complete design was performed in random order, involving 17 experiments, including 5 replicates at the central point (Table 1). The analyses were determined by the following formula:

$$
Y=\beta_{0}+\sum b_{i} X_{i}+\sum b_{i i} X_{i}^{2}+\sum b_{i j} X_{i} X_{j}
$$

The quality of the fitted model was determined by the coefficient of determination (R). A central composite design (CCD) was carried out to study the effects of the three independent variables.

\subsection{Proximate Composition}

Samples were evaluated using AOAC (The Association of Official Analytical Chemists) procedures with minor modifications [21]. The total carbohydrate content was measured by subtracting the contents of moisture, ash, fat, and protein from 100 and reported as a percentage of dry mass. The dietary fiber content was determined by enzymatic and gravimetric methods. The crude protein content was evaluated by the Macro-Kjeldahl method.

\subsection{Macro- and Microelements}

Determination of the mineral content (ash) and analysis of the mineral elements were made on dry samples via the AOAC method no. 930.05. The incineration residue was extracted with $0.5 \mathrm{~mL} / \mathrm{mL} \mathrm{HCl}$ and $0.5 \mathrm{~mL} / \mathrm{mL} \mathrm{HNO}_{3}$, then distilled water was added and $\mathrm{Fe}, \mathrm{Cu}, \mathrm{Mn}$, and $\mathrm{Zn}$ were weighed directly $[22,23]$. Standard solutions were used to compare absorption responses with standard analytical solutions of purity $>99.9 \%$.

\subsection{Reducing Power}

The reducing power of the ultrasonic extract was determined according to an established method [24]. Increased absorbance of the reaction mixture indicated increased reducing power. Evaluation of the reduction potency was performed by mixing each extract in different solutions. The mixture was incubated at $50{ }^{\circ} \mathrm{C}$ for $20 \mathrm{~min}$. Trichloroacetic acid (TCA, 10\%, $2.5 \mathrm{~mL}$ ) was added to a portion of this mixture $(5 \mathrm{~mL})$ and centrifuged at $3000 \times g$ for $10 \mathrm{~min}$. The supernatant was separated and mixed with distilled water $(2.5 \mathrm{~mL})$ containing $1 \%$ ferric chloride $(0.5 \mathrm{~mL})$. The absorbance was measured at $700 \mathrm{~nm}$ (Ocean Optics HR2000+ Spectrometer, Ostfildern, Germany).

\subsection{Determination of the Total Phenol and Flavonoid Contents of H. erinaceus Extract}

The flavonoid content was measured according to the method described by Delcour and Varebeke [25], and quercetin was used for the analytical curve. The results are expressed as milligrams 
of quercetin equivalent per gram of dry matter of mushroom extract (mg QE/g DM). After an incubation period of $10 \mathrm{~min}$, the absorbance was read at $640 \mathrm{~nm}$. The total phenolic contents of the extracts were calculated using Folin-Ciocalteu reagent, following the method of Velioglu [26], where gallic acid (mg GAE/g DM) was used as a standard antioxidant. The analysis was performed using an Ocean Optics HR2000+ Spectrometer (Ostfildern, Germany).

\subsection{DPPH-Radical Scavenging (Antioxidant) Activity}

The free radical scavenging rate was determined by measuring the 2,2-diphenyl-1-picrylhydrazyl $(\mathrm{DPPH})$ with L-ascorbic acid as a reference standard. For the DPPH assay, we used a modified procedure described in a previous study [27]. The lyophilized powder extract from Hericium erinaceus was dissolved in methanol ( $2 \mathrm{~mL}$ extracts and $2 \mathrm{~mL}$ Tris- $\mathrm{HCl}$ buffer) and mixed with $260 \mu \mathrm{L}$ of a $0.2 \mathrm{mM}$ DPPH radical solution (Sigma-Aldrich, St. Louis, MO, USA). The percentage inhibition was calculated according to the formula $\left[\left(\mathrm{A}_{0}-\mathrm{A}_{1}\right) / \mathrm{A}_{0}\right] \times 100$, where $\mathrm{A}_{0}$ is the absorbance of the control and $\mathrm{A}_{1}$ is the absorbance of the sample.

\subsection{Determination of Acetylcholinesterase (AChE) and Butyrylcholinesterase (BChE) Inhibitory Activities}

The AChE and BChE inhibitory activities were measured by slightly modifying the spectrophotometric method [28]. Electric eel AChE (Type-VI-S, EC 3.1.1.7, Sigma Aldrich/MERK, Milan, Italy) and horse serum BChE (Sigma Aldrich/MERK, Milan, Italy) were used, while acetylthiocholine iodide and butyrylthiocholine chloride (Sigma Aldrich/MERK, Milan, Italy) were chosen as the substrates of the reaction. Galantamine (Sigma Aldrich/MERK, Milan, Italy), was used as the reference drug [29].

\subsection{HPLC/DAD-UV Analysis}

HPLC analysis of erinacine A was conducted according to a previous study [18]. The retention time of erinacine A was approximately $\sim 17.8 \mathrm{~min}$ at a flow rate of $1.0 \mathrm{~mL} / \mathrm{min}$ at $380 \mathrm{~nm}$. Erinacine A was used as a standard as previously reported [30]. A stock solution $(1 \mathrm{mg} / \mathrm{mL})$ of erinacine A was prepared in $70 \%$ ethanol. Standard solutions of erinacine A within the final concentration range of $1-25 \mu \mathrm{g} / \mathrm{mL}$ were prepared (see Figure S1 for the calibration curve).

\subsection{Statistical Analysis}

The experimental results of the RSM were analyzed using Matlab2018b software. $p$-Values less than 0.05 were considered to indicate statistical significance. The data were analyzed using GraphPad Prism 7 software, and the HPLC data were analyzed using OriginPro software.

\section{Results}

\subsection{Effect of Different Ultrasonic Parameters on the Yield of H. erinaceus Extracts}

A microscopic analysis (Olympus BX 43, Hamburg, Germany) was performed to confirm the development of $H$. erinaceus biomass in the culture vessel and observe the presence of mycelial hyphae (Figure 2), and images were taken with the digital camera of the Olympus Bx 43 instrument. The analysis was performed with white LED lamp housing (U-LHLEDC), with a 40x objective lens and a 10-fold ocular, using an Olympus DP72 camera sensor (Olympus Corporation, Hamburg, Germany) (11.8 megapixel cooled digital color camera, capturing each color of RGB at 12 bits, at ISO 1600 setting, image color-calibrated (XC30 software)). The parameters tracked during ultrasonic extraction by sonochemistry were approximately (extraction time being different) similar for each sample examined and were constantly monitored from the operating system of the device using $27 \mathrm{~W}$ per hour, with a net extraction power of $170 \mathrm{~W}$, and maintaining a constant temperature in the ultrasonic bath. 


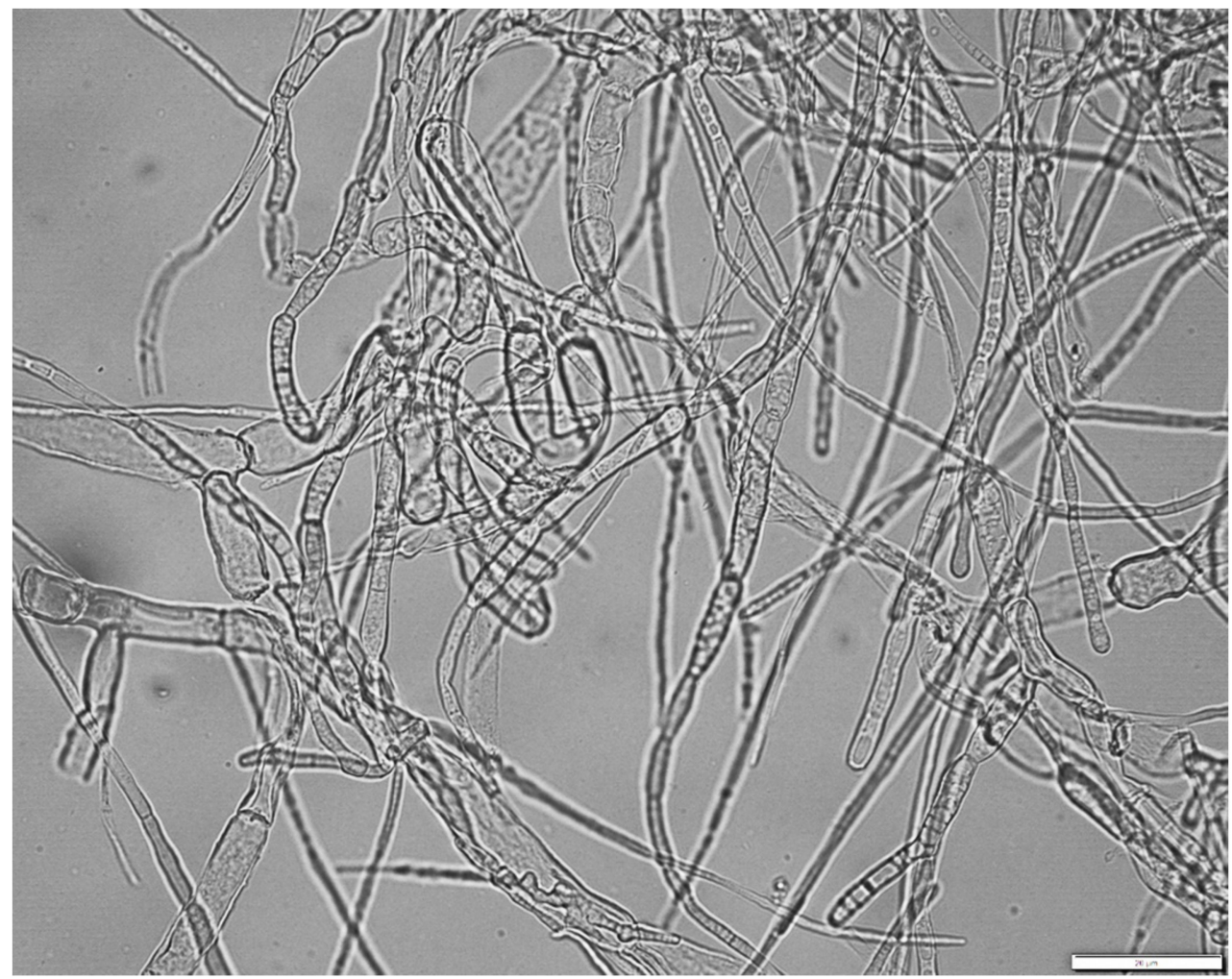

Figure 2. Mycelial filamentous hyphae developed inside the biomass of Hericium erinaceus (Olympus BX43, Hamburg, Germany). Scale bar: $20 \mu \mathrm{m}$.

It should be specified that temperature and time were controlled in order not to degrade biological compounds in the H. erinaceus biomass, and the former was maintained constant by circulating external cold water and using a T-type thermocouple. However, the temperature of the ultrasonic bath during the entire sonication period was kept as constant as possible by the addition of ice sheets. In general, ultrasonication increases the rate of a chemical reaction; the reaction conditions are easier to meet, and the energy consumed can be reduced [10]. To achieve a more exhaustive procedure, it was important to investigate the process variables. Preliminary experiments enabled the range of ethanol concentrations (40-80\%), extraction time (20-45 $\mathrm{min})$, and solvent-to-material ratio $(10-30 \mathrm{~mL} / \mathrm{g}$ ) to be fixed. It can be seen in Tables 1 and 2 that the extraction yield was affected most significantly by ethanol $\left(\mathrm{X}_{1}\right)(p<0.05)$ and the solvent-to-material ratio $\left(\mathrm{X}_{3}\right)$, followed by extraction time $\left(\mathrm{X}_{2}\right)(p<0.05)$. The quadratic parameter $\mathrm{X}_{1}^{2}$ was significant at the level of $p<0.05$, whereas the two quadratic parameters $\left(X_{2}^{2}, X_{3}^{2}\right)$ and interaction quadratic parameters $\left(X_{1} X_{2}, X_{2} X_{3}, X_{1} X_{3}\right)$ were non-significant $(p>0.05)$ with respect to extraction yield. 
Table 1. Box-Behnken Design (BBD) experimental design with the independent variables and observed responses.

\begin{tabular}{|c|c|c|c|c|c|c|c|}
\hline Run Order ${ }^{a}$ & $\begin{array}{l}\text { Ethanol\% } \\
\qquad\left(X_{1}\right)\end{array}$ & $\begin{array}{l}\text { Extraction Time } \\
\qquad\left(X_{2}\right)(\mathrm{min})\end{array}$ & $\begin{array}{l}\text { Solvent-to-Material Ratio } \\
\qquad\left(\mathrm{X}_{3}\right)(\mathrm{mL} / \mathrm{g})\end{array}$ & Yield\% & $\begin{array}{c}\text { TPC }^{\mathrm{b}} \\
\text { (mg GAE/g DM) }\end{array}$ & $\begin{array}{c}\mathrm{TFC}^{\mathrm{b}} \\
\text { (mg QE/g DM) }\end{array}$ & $\begin{array}{l}\text { DPPH Assay } \\
\text { IC }_{50}(\mu \mathrm{g} / \mathrm{mL})\end{array}$ \\
\hline 1 & 40 & 20 & 20 & 9.30 & $11.1 \pm 0.56$ & $1.98 \pm 0.01$ & $55.3 \pm 0.32$ \\
\hline 2 & 80 & 20 & 20 & 15.20 & $21.2 \pm 0.65$ & $3.11 \pm 0.01$ & $85.4 \pm 0.15$ \\
\hline 3 & 40 & 45 & 20 & 10.50 & $13.3 \pm 0.32$ & $1.88 \pm 0.01$ & $56.3 \pm 0.27$ \\
\hline 4 & 80 & 45 & 20 & 17.50 & $22.2 \pm 0.29$ & $3.25 \pm 0.01$ & $92.4 \pm 0.37$ \\
\hline 5 & 40 & 30 & 10 & 9.60 & $12.8 \pm 0.18$ & $1.20 \pm 0.01$ & $49.3 \pm 0.29$ \\
\hline 6 & 80 & 30 & 10 & 16.20 & $22.3 \pm 0.33$ & $2.65 \pm 0.01$ & $88.2 \pm 0.21$ \\
\hline 7 & 40 & 30 & 30 & 9.20 & $11.1 \pm 0.14$ & $0.65 \pm 0.01$ & $51.6 \pm 0.36$ \\
\hline 8 & 80 & 45 & 30 & 18.00 & $23.2 \pm 0.11$ & $3.26 \pm 0.01$ & $87.2 \pm 0.77$ \\
\hline 9 & 60 & 20 & 10 & 13.30 & $13.5 \pm 0.65$ & $0.96 \pm 0.01$ & $65.1 \pm 0.54$ \\
\hline 10 & 60 & 45 & 10 & 13.20 & $14.2 \pm 0.54$ & $0.85 \pm 0.01$ & $61.1 \pm 0.11$ \\
\hline 11 & 60 & 20 & 30 & 13.10 & $14.1 \pm 0.64$ & $0.75 \pm 0.01$ & $63.8 \pm 0.91$ \\
\hline 12 & 60 & 45 & 30 & 12.90 & $13.2 \pm 0.26$ & $0.84 \pm 0.01$ & $59.2 \pm 0.46$ \\
\hline 13 & 60 & 30 & 20 & 12.90 & $13.6 \pm 0.45$ & $0.59 \pm 0.01$ & $52.1 \pm 0.61$ \\
\hline 14 & 60 & 30 & 20 & 12.60 & $13.2 \pm 0.19$ & $0.59 \pm 0.01$ & $52.6 \pm 0.45$ \\
\hline 15 & 60 & 30 & 20 & 12.60 & $12.6 \pm 0.57$ & $0.61 \pm 0.01$ & $49.8 \pm 0.32$ \\
\hline 16 & 60 & 30 & 20 & 12.50 & $13.2 \pm 0.75$ & $0.61 \pm 0.01$ & $52.6 \pm 0.41$ \\
\hline 17 & 60 & 30 & 20 & 12.50 & $13.6 \pm 0.94$ & $0.59 \pm 0.01$ & $52.8 \pm 0.67$ \\
\hline
\end{tabular}

${ }^{a}$ Run order-randomized. ${ }^{b}$ The values are given as mean \pm standard deviation of triplicate determinations; total phenolic content (TPC) expressed as mg GAE/g DM; total flavonoid content (TFC) expressed as mg QE/g DM; free radical scavenging activity expressed as $\mu \mathrm{g} / \mathrm{mL}$; DM: dry matter. 
Table 2. Estimated regression model of the relationships among response variables (yield, TPC, TFC, and DPPH of $H$. erinaceus extracts) and independent variables $\left(X_{1}, X_{2}, X_{3}\right)$.

\begin{tabular}{ccccc}
\hline Variable & \multicolumn{3}{c}{$p$-Value } \\
\hline & Yield & TPC & TFC & DPPH \\
\hline$\beta_{0}$ & $<0.001$ & $<0.001$ & $>0.001$ & $<0.001$ \\
$X_{1}$ & $<0.001$ & $<0.001$ & $>0.001$ & $<0.001$ \\
$X_{2}$ & 0.0352 & $<0.001$ & $>0.001$ & $<0.001$ \\
$X_{3}$ & $<0.001$ & $<0.001$ & $>0.001$ & $<0.001$ \\
$X_{1}{ }^{2}$ & 0.0312 & $<0.001$ & $>0.001$ & $<0.001$ \\
$X_{2}{ }^{2}$ & 0.217 & $<0.001$ & $>0.001$ & $<0.001$ \\
$X_{3}$ & 0.281 & $<0.001$ & $>0.001$ & $<0.001$ \\
$X_{1} X_{2}$ & 3.36 & $<0.001$ & $>0.001$ & $<0.001$ \\
$X_{1} X_{3}$ & 10.3 & $<0.001$ & $>0.001$ & $<0.001$ \\
$X_{2} X_{3}$ & 12.5 & $<0.001$ & $>0.001$ & $<0.001$ \\
\hline
\end{tabular}

\subsection{Response Surface Method (RSM)}

Figure 3 displays the interactions among the ethanol concentration and each of the two other factors, namely, extraction time and solvent-to-material ratio; the effect of their mutual interaction on the extraction yield of $H$. erinaceus can thus be extrapolated.

In Figure $3 \mathrm{~A}$ is shown the amplitude of the interaction between ethanol concentration $\left(\mathrm{X}_{1}\right)$ and extraction time $\left(\mathrm{X}_{2}\right)$ regarding the extraction yield. Increasing percentages of ethanol from 60 to $80 \%$ with extraction time from 30 to 45 min enhanced the extraction yield. It can be seen in Figure 3B that by varying the ethanol concentration from 60 to $80 \%$ and increasing the solvent-to-material ratio from 10 to $30 \mathrm{~mL} / \mathrm{g}$, the extraction yield of target compounds was improved. Figure $3 \mathrm{C}$ presents the correlation between extraction time and the solvent-to-material ratio. It was found that the maximum yield $(17.50 \%)$ was achieved when the extraction time was $45 \mathrm{~min}$ and the solvent-to-material ratio was $20 \mathrm{~mL} / \mathrm{g}$. This result was in accordance with the findings of previous studies [31,32].

\subsection{Effect of Extraction Parameters on TPC, TFC, and DPPH}

The total phenolic content in the extract is expressed in milligrams of gallic acid equivalent and the total flavonoid content in milligrams of quercetin equivalent. In this study, however, the total phenolics yield decreased when the ethanol concentration was more than $80 \%$ (Table 1 ). The total phenolics yield increased with prolonged extraction time from 20 to $45 \mathrm{~min}$. The total phenolics yield increased with an increase in ethanol concentration from $60 \%$ to $80 \%$. It should be noted that, although in a very small amount, flavonoids are also present in the extract of $H$. erinaceus. The ethanol extracts exhibited high content of phenolics and high radical scavenging activity (DPPH scavenged, as a percentage). The results strongly suggest that phenolics and flavonoids are important components of the H. erinaceus extracts, and this could explain their high radical scavenging activity. The total flavonoid (TFC) and phenolic (TPC) contents of H. erinaceus were quantified using the Folin-Ciocalteu reagent (Table 1). It was found that the total phenolic content of $H$. erinaceus reached $23.26 \mathrm{mg}$ GAE/g DM and the total flavonoid content reached $3.26 \mathrm{mg}$ QE/g DM with $80 \%$ ethanol concentration, extraction time of $45 \mathrm{~min}$, and solvent-material ratio $30 \mathrm{~mL} / \mathrm{g}$, these data being consistent with those from other scientific studies [12,33]. In the DPPH assay, the maximum antioxidant activity of H. erinaceus corresponded to an $\mathrm{IC}_{50}$ of $92.4 \mu \mathrm{g} / \mathrm{mL}$. The results of the present study showed that a dose-dependent increase in free radical extinction is due to the increase in alcohol concentration $(80 \%)$ with the lowest $\mathrm{IC}_{50}$ value in this assay, as shown in Table 1. 


\subsection{Antioxidant Capacities of H. erinaceus Ultrasonic Extracts Concerning Total Phenolics and Other Compounds}

The phenols in mushroom extracts can be linked to their antioxidant capabilities. Our research highlights a correlation between total phenolics and DPPH free radical scavenging activity in mushroom extracts. This is consistent with the research of several authors who reported that the total phenolic content correlated with the free radical scavenging activity of other fungi $[34,35]$. As determined by biochemical methods, the antioxidant activity expressed by the percentage inhibition of the lipid peroxidation reaction was the highest in $H$. erinaceus $80 \%$ ethanolic extract. The results confirmed a correlation between these remarkable antioxidant activities and a high content of compounds with free radical scavenging properties.

\subsection{Proximate Composition}

The results of the quantitative analyses to determine the percentages of protein, fat, fiber, carbohydrate, and ash in the bioreactor-developed H. erinaceus mycelia are reported in Table 3.

Table 3. Proximate composition ( $\mathrm{g} / 100 \mathrm{~g} \mathrm{dw})$, free sugars, and fatty acids in H. erinaceus.

\begin{tabular}{lcccccccc}
\hline Mushroom & $\begin{array}{c}\text { Crude } \\
\text { Protein (\%) }\end{array}$ & $\begin{array}{c}\text { Crude } \\
\text { Fat (\%) }\end{array}$ & $\begin{array}{c}\text { Fiber } \\
\mathbf{( \% )}\end{array}$ & $\begin{array}{c}\text { Carbohydrate } \\
\mathbf{( \% )}\end{array}$ & $\begin{array}{c}\text { Arabinose } \\
\mathbf{( \% )}\end{array}$ & $\begin{array}{c}\text { Mannitol } \\
\mathbf{( \% )}\end{array}$ & $\begin{array}{c}\text { Ash } \\
\mathbf{( \% )}\end{array}$ & $\begin{array}{c}\text { Energy } \\
\text { Kcal/100 g (\%) }\end{array}$ \\
\hline H. erinaceus & $15.04 \pm 0.20$ & $2.03 \pm 0.40$ & $6.12 \pm 0.20$ & $77.23 \pm 0.30$ & $16.46 \pm 0.20$ & $5.26 \pm 0.20$ & $6.53 \pm 0.20$ & $375.30 \pm 0.10$ \\
\hline
\end{tabular}

Values are expressed as mean \pm SD of carefully conducted triplicate experiments.

\subsection{Macro- and Microelements}

The mineral composition usually reflects the growth conditions of the mycelia. In other scientific articles, it has been found that medicinal mushrooms contain many mineral elements [16,36]. Our results are highlighted in Table 4. Other researchers mentioned that $\mathrm{K}$ is the main macroelement in mushrooms [37], and this was also found in our research.

Table 4. Macro- and microelements in the Hericium erinaceus biomass developed in the bioreactor.

\begin{tabular}{cc}
\hline Macroelements $\mathbf{( m g / 1 0 0 ~} \mathbf{g} \mathbf{d w})$ & Hericium erinaceus \\
\hline $\mathbf{C a}$ & $42.32 \pm 2.36$ \\
$\mathbf{M g}$ & $74.54 \pm 3.14$ \\
$\mathbf{N a}$ & $546.23 \pm 3.10$ \\
$\mathbf{K}$ & $1030.03 \pm 12.10$ \\
\hline Microelements $\mathbf{( m g / 1 0 0} \mathbf{g ~ d w})$ & \\
\hline $\mathbf{F e}$ & $5.23 \pm 0.02$ \\
$\mathbf{C u}$ & $0.14 \pm 0.02$ \\
$\mathbf{M n}$ & $0.04 \pm 0.01$ \\
$\mathbf{Z n}$ & $2.13 \pm 0.03$ \\
\hline
\end{tabular}

\subsection{Reducing Power}

The formation was monitored spectrophotometrically at $700 \mathrm{~nm}$. In this study, the scavenging effect of $H$. erinaceus was limited and was found to be inferior, even at higher extract concentration, to that of three standard antioxidants (Table 5).

The investigated mushroom dry extract possessed reductive capabilities, although inferior to ascorbic acid, quercetin, and butylated hydroxyanisole, used as standard antioxidants at the same concentration $(0.50 \mathrm{mg} / \mathrm{mL})$. The reducing powers of the extracts produced by ultrasonication with ethanol solvent gave high levels of absorbance. Thus, discrete levels of antioxidant compounds were highlighted. 

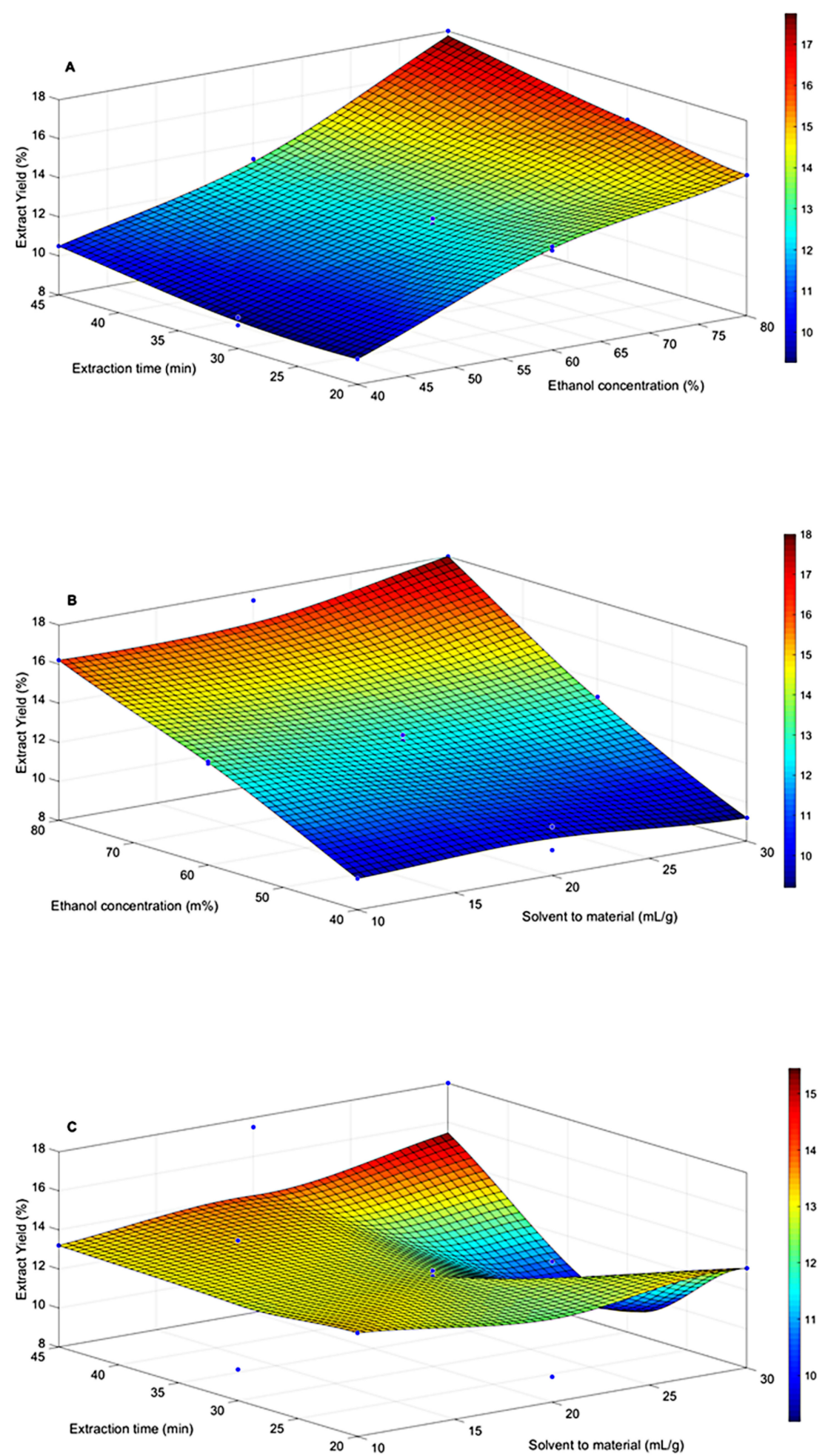

Figure 3. Response surface analysis for the extract yield (\%) via ultrasonic extraction (UE) for (A) extraction time and ethanol concentration; (B) ethanol concentration and solvent-to-material ratio; (C) extraction time and solvent-to-material ratio. The data were analyzed using Matlab software. 
Table 5. Reducing power of Hericium erinaceus extract.

\begin{tabular}{|c|c|c|c|c|}
\hline & \multicolumn{4}{|c|}{ Absorbance Values (at $700 \mathrm{~nm})$ at Different Concentrations $(\mathrm{mg} / \mathrm{mL})$} \\
\hline & 0.05 & 0.10 & 0.50 & 1.00 \\
\hline H. erinaceus extract & $0.020 \pm 0.004$ & $0.024 \pm 0.021$ & $0.021 \pm 0.006$ & $0.065 \pm 0.015$ \\
\hline Quercetin $^{\mathrm{a}}$ & & & $2.501 \pm 0.052$ & \\
\hline Butylated hydroxyanisole (BHA) ${ }^{a}$ & & & $2.401 \pm 0.016$ & \\
\hline Ascorbic acid ${ }^{a}$ & & & $2.126 \pm 0.127$ & \\
\hline
\end{tabular}

\subsection{AChE and BChE Inhibitory Activity}

Because of the previous experiments that revealed high antioxidant activity of Hericium erinaceus extract obtained using an ethyl alcohol concentration of $80 \%$, we wanted to further check the possible effect on acetylcholinesterase and butyrylcholinesterase to achieve multitarget action for the treatment of AD. Table 6 reports the levels of acetylcholinesterase and butyrylcholinesterase after treatment with the H. erinaceus extracts compared with those for galantamine. As shown in Table 6, all extracts showed mild butyrylcholinesterase and acetylcholinesterase inhibitory activities. The $80 \%$ alcohol extracts of H. erinaceus demonstrated higher inhibitory activity, giving inhibition percentages of up to $53 \%$ against AChE and $49 \%$ against BChE at $1 \mathrm{mg} / \mathrm{mL}$.

Table 6. Acetylcholinesterase and butyrylcholinesterase inhibitory activity levels of the $80 \%$ ethanolic H. erinaceus extracts.

\begin{tabular}{|c|c|c|c|}
\hline Run Order & $\begin{array}{l}\text { Extraction Time } \\
\text { min }\end{array}$ & $\begin{array}{l}\text { AChE Inhibition \% } \\
\quad(1 \mathrm{mg} / \mathrm{mL})^{a}\end{array}$ & $\begin{array}{c}\text { BChE Inhibition \% } \\
(1 \mathrm{mg} / \mathrm{mL})^{a}\end{array}$ \\
\hline 1 & 20 & $51 \pm 1.5$ & $48 \pm 1.4$ \\
\hline 2 & 30 & $52 \pm 1.4$ & $48 \pm 2.5$ \\
\hline 3 & 45 & $53 \pm 2.5$ & $49 \pm 3.1$ \\
\hline Galantamine & - & $73 \pm 2.6$ & $62 \pm 3.2$ \\
\hline
\end{tabular}

a Inhibition percentages represent the mean \pm standard deviation of three parallel measurements $(p<0.05)$.

\subsection{HPLC/DAD-UV Analysis}

Erinacine A was obtained in the glucose-containing medium, as described by [38]. Our work describes the validation of an HPLC/DAD-UV method for the determination of the biocompound erinacine A from the extract of Hericium erinaceus (solid-state cultivation) obtained by ultrasonic extraction. The representative HPLC chromatogram of the powder extract of H. erinaceus mycelia is shown in Figure 4. The retention time at 17.846 represents erinacine A, one of the most characteristic compounds found in general in Hericium species [39]. 




Figure 4. The HPLC sample chromatogram of erinacine A (obtained from H. erinaceus mycelium powder extract). The retention time of the diterpenoid erinacine A peak was within the range of 17.846-17.852 $\mathrm{min}$.

\section{Discussion}

Past research has shown that the biological activity of fungi can be attributed to polysaccharides, high-molecular-weight glucans [40], and high contents of polyphenols and flavonoids, which are responsible for cytotoxic and antioxidant activities [41,42]. In order to improve the production and the recovery of such bioactive compounds, as well as the content of erinacine A, we modified the development conditions of $H$. erinaceus mycelia in a bioreactor. Then, we used ultrasonication to improve the efficiency of extraction. It is important to also note the fact that not all extracts could furnish a dry product in powder form [20]. Some extracts, particularly non-polar extracts, might lead to an oily product, so consideration should be given to optimizing the extraction method. In our experiments, we can state that our procedure led to a powdered fungal material that maintained and preserved the analyzed biocompounds, as well as the valuable contents of micro- and macroelements. It is known that the lyophilization technique maintains the highest amount of antioxidant compounds compared to other methods of drying extracts. These results were confirmed by previously published studies that showed that the lyophilization process is the most effective way to maintain a high amount of flavonoids in fungi [35]. The differences from other authors [15,16] were likely due to the different conditions of development in the bioreactor of the biological material and the extraction process with ultrasonication.

Given the follow-up, it can be said that a large amount of $H$. erinaceus powder was obtained from a small amount of fungal material. Also, the improvement of the extraction of polyphenols and flavonoids by ultrasonication can mainly be attributed to the effect of acoustic cavitations produced in the solvent by the passage of ultrasonic waves. Therefore, the structure of the cell wall is interrupted, and diffusion through the membranes is accelerated. The most efficient extraction period for achieving the maximum yield was approximately $45 \mathrm{~min}$. However, other parameters could be considered for 
optimizing the extraction yields of diterpenoids and polyphenols, such as the ultrasonic amplitude of the precipitation time. Based on our results, we can say that the H. erinaceus mycelia developed in the culture vessel after our optimized process could improve the extraction of polyphenols, flavonoids, and diterpenoids. This can also be attributed to ultrasonic extraction.

The ANOVA outcome on our extraction conditions, shown in Table 2, revealed that the first-order terms of independent variables $\left(X_{1}, X_{2}\right.$, and $\left.X_{3}\right)$, quadratic terms $\left(X_{1}{ }^{2}, X_{3}{ }^{2}\right.$, and $\left.X_{2}{ }^{2}\right)$, and interaction terms $\left(X_{1} X_{2}, X_{1} X_{3}\right.$, and $\left.X_{2} X_{3}\right)$ mostly affected the content of total phenolics recovered for $H$. erinaceus $(p<0.05)$. This aspect can be explained by the prolongation of the extraction time, which accelerates the chemical decomposition of the bioactive compounds in the extraction process, resulting in lower extraction efficiency. Multiple regression analysis was performed on the data of response variables, such as the DPPH, TPC, and TFC content, as affected by the extraction conditions. Other authors [43-47] also documented positive correlations between total phenolics and DPPH radical scavenging activity, but also the antioxidant potential of mushrooms, due to the presence of the diterpenoid erinacine A. The results indicate that healthy ingredients can be maximally extracted using the optimal solvents and parameters for mushroom extractions, thus modulating the difference in pharmacological effects. Indeed, our results indicated that there was a correlation between DPPH inhibitory activity and total phenolics due to the application of ultrasonic extraction. Thus, from this experiment we concluded that $H$. erinaceus can control oxidative damage and can act as an antioxidant.

The ethanol extracts exhibited high contents of phenolics and high radical scavenging activity (DPPH scavenged, as a percentage). The results strongly suggest that phenolics and flavonoids are important components of the H. erinaceus extracts, and this could explain their high radical scavenging activity. Extracts of $H$. erinaceus are free radical inhibitors or scavengers, acting possibly as primary antioxidants. It has been reported that antioxidant effects of a compound may be concomitant with the development of reducing power. Reducing properties are generally associated with the presence of reductones, which have been shown to exert antioxidant action by breaking the free radical chain via the donation of a hydrogen atom [48]. Conversely, in the DPPH radical investigation, the results suggested that some other antioxidant compounds, not only total phenolics, are responsible for reductive capabilities. Overall, from this analysis we concluded that $H$. erinaceus can control oxidative damage and can act as an antioxidant. Ultrasonic extraction of $H$. erinaceus with ethanol resulted in relatively good antioxidant activity concerning the extracts described in these previous reports. Accordingly, Gursoy et al. [49] noted that the radical scavenging activity on DPPH of methanol extracts from $M$. rotunda at $2 \mathrm{mg} / \mathrm{mL}$ was $33.94 \pm 0.96 \%$. The variation of the antioxidant activity in many scientific articles can also be attributed to the solvent used [50].

Moreover, our results are in accordance with literature data for other Hericium species [44]. HPLC analysis showed erinacine A as the main compound in our ultrasonic-derived alcoholic extracts, suggesting that it may also be responsible for its antioxidant effects. The yield rate of erinacine A in the H. erinaceus after ethanol extraction was $\sim 4 \mathrm{mg} / \mathrm{kg}$, as confirmed and quantified by the HPLC procedure [18].

The chemical composition of mushrooms varies according to species [51,52]. Among the Hericium spp., H. erinaceus is the most studied. Its fruiting body is known as a good source of carbohydrates (76.5\% DW, dry weight), protein (18.8\% DW), ash (7.52\% DW), fiber (7.10\% DW), and fat (2.01\% DW). These mushrooms also contain several amino acids, substantial amounts of potassium and phosphorus, and aroma substances [52]. The proximal composition of Hericium erinaceus can be seen in Table 3 . H. erinaceus contains high energy, proteins, total antioxidant capacity (TAC), fiber, and fat. In the literature, arabinose has been evaluated as one of the minor sugars in mushrooms [36], but for the $H$. erinaceus studied herein, arabinose was the major sugar, and this can be attributed to the protocol followed for the development of the H. erinaceus mycelia. A large amount of arabinose is a characteristic of the genus Hericium $[53,54]$. The nutritional composition of $H$. erinaceus biomass can also be related to the composition of fat, protein, and carbohydrates used for cultivation in the glass vessel. 
However, the inhibitory activity of each ethanol extract of H. erinaceus toward AChE was significantly $(p<0.001)$ lower than that of galantamine, the positive control. A previous study reported that the percentage of acetylcholinesterase inhibition activity in the fungal species Pleurotus pulmonarius ranged from 57.24 to $69.05 \%$ at concentrations of $0.063-1.0 \mathrm{mg} / \mathrm{mL}$ [48].

Diverse studies have reported that edible mushrooms possess bioactive compounds that enhance various antioxidant enzymes in the body [1]. Over the past decade, $H$. erinaceus has attracted increasing attention in the fields of functional foods and biomedicine. Numerous studies have reported the potential medicinal values or health-beneficial activities of this mushroom, like anti-cancer, anti-hypertensive, hypolipidemic, and neuronal disease protecting activities $[1,11]$. This study also established a good relationship between radical scavenging activity and phenolic/flavonoid compounds, thus suggesting a potential neuroprotective effect in AD.

\section{Conclusions}

This is the first study to investigate the antioxidant potential of Hericium erinaceus extracts obtained by ultrasonic extraction with ethanol. Based on our study, we can conclude that mushrooms seem to be a potential natural source of dietary flavonoids and polyphenols, displaying a great range of compounds in valuable concentrations. These results indicate the medicinal and antioxidant properties of H. erinaceus for use in alternative medicine. The methodology realized the development of H. erinaceus biomass in a controlled in vitro medium and its subsequent submission to the ultrasonic extraction technique, which can enrich the isolation of antioxidants in Hericium erinaceus, particularly polyphenols and flavonoids correlated with the diterpenoid erinacine A, known for its high antioxidant activity. The optimized extraction conditions were $80 \%$ ethanol, extraction time of $45 \mathrm{~min}$, and solvent-material ratio of 1:30 $(\mathrm{g} / \mathrm{mL})$. The total content of phenolics in this optimized H. erinaceus extract was $23.2 \mathrm{mg}$ GAE/g DM, and in the DPPH test, the antioxidant activity reached an $\mathrm{IC}_{50}$ of $87.2 \mu \mathrm{g} / \mathrm{mL}$. According to the results above, $H$. erinaceus biomass may be introduced as a possible valuable source of nutritive supplements exhibiting antioxidant properties. Our future studies are ongoing and will focus on researching the possible beneficial effects on the brain of $H$. erinaceus extracts.

Supplementary Materials: The following are available online at http://www.mdpi.com/2304-8158/9/12/1889/s1, Figure S1: Erinacine A calibration curve for the HPLC method.

Author Contributions: Conceptualization, S.C. and M.-V.V.; methodology, L.C.S. and N.A.S.; formal analysis, C.D., S.M., L.H. and R.S.B.; investigation, S.C., L.C.S., N.A.S. and C.D.; resources, C.D. and S.M.; writing and original draft preparation, S.C., L.H., C.D. and M.-V.V. All authors have read and agreed to the published version of the manuscript.

Funding: This research was funded by a grant from the Romanian National Authority for Scientific Research and Innovation, CCCDI-UEFISCDI, project number PN-III-P1-1.2-PCCDI-2017-0662, contract 12PCCDI/2018 to C.D. The APC was funded by S.C. and M.-V.V.

Acknowledgments: We thank CS III. Denis Negrea for critically reviewing the manuscript and for the English editing of the manuscript.

Conflicts of Interest: The authors declare no conflict of interest.

\section{References}

1. Blumfield, M.; Abbott, K.; Duve, E.; Cassettari, T.; Marshall, S.; Fayet-Moore, F. Examining the health effects and bioactive components in Agaricus bisporus mushrooms: A scoping review. J. Nutr. Biochem. 2020, 84, 108453. [CrossRef] [PubMed]

2. Limanaqi, F.; Biagioni, F.; Mastroiacovo, F.; Polzella, M.; Lazzeri, G.; Fornai, F. Merging the multi-target effects of phytochemicals in neurodegeneration: From oxidative stress to protein aggregation and inflammation. Antioxidants 2020, 9, 1022. [CrossRef] [PubMed]

3. Chandran, R.; Abrahamse, H. Identifying plant-based natural medicine against oxidative stress and neurodegenerative disorders. Oxid. Med. Cell. Longev. 2020, 2020, 8648742. [CrossRef] [PubMed] 
4. Uddin, M.S.; Al Mamun, A.; Kabir, M.T.; Ahmad, J.; Jeandet, P.; Sarwar, M.S.; Ashraf, G.M.; Aleya, L. Neuroprotective role of polyphenols against oxidative stress-mediated neurodegeneration. Eur. J. Pharmacol. 2020, 886, 173412. [CrossRef]

5. Crous-Bou, M.; Minguillon, C.; Gramunt, N.; Molinuevo, J.L. Alzheimer's disease prevention: From risk factors to early intervention. Alzheimer Res. Ther. 2017, 9, 71. [CrossRef]

6. Gupta, A.; Naraniwal, M.; Kothari, V. Modern extraction methods for preparation of bioactive plant extracts. IJANS 2012, 1, 8-26.

7. Rollinger, J.M.; Stuppner, H.; Langer, T. Virtual screening for the discovery of bioactive natural products. In Natural Compounds as Drugs Volume I; Progress in Drug, Research; Petersen, F., Amstutz, R., Eds.; Birkhäuser: Basel, Switzerland, 2008. [CrossRef]

8. Gong, P.; Wang, S.; Liu, M.; Chen, F.; Yang, W.; Chang, X.; Liu, N.; Zhao, Y.; Wang, J.; Chen, X. Extraction methods, chemical characterizations and biological activities of mushroom polysaccharides: A mini-review. Carbohydr. Res. 2020, 494, 108037. [CrossRef]

9. Lianfu, Z.; Zelong, L. Optimization and comparison of ultrasonic/microwave assisted extraction (UMAE) and ultrasonic assisted extraction (UAE) of lycopene from tomatoes. Ultrason. Sonochem. 2008, 15, 731-737. [CrossRef]

10. Gallo, M.; Ferrara, L.; Naviglio, D. Application of ultrasonic in food science and technology: A perspective. Foods 2018, 7, 164. [CrossRef]

11. Wang, M.; Gao, Y.; Xu, D.; Konishi, T.; Gao, Q. Hericium erinaceus (Yamabushitake): A unique resource for developing functional foods and medicines. Food Funct. 2014, 5, 3055-3064. [CrossRef]

12. Gąsecka, M.; Mleczek, M.; Siwulski, M.; Niedzielski, P.; Kozak, L. Phenolic and flavonoid content in Hericium erinaceus, Ganoderma lucidum, and Agrocybe aegerita under selenium addition. Acta Aliment. 2016, 45, 300-308. [CrossRef]

13. Lew, S.Y.; Yow, Y.Y.; Lim, L.W.; Wong, K.H. Antioxidant-mediated protective role of Hericium erinaceus (Bull.: Fr.) Pers. against oxidative damage in fibroblasts from Friedreich's ataxia patient. Food Sci. Technol. 2020, 40, 264-272. [CrossRef]

14. Jiang, S.; Wang, S.; Sun, Y.; Zhang, Q. Medicinal properties of Hericium erinaceus and its potential to formulate novel mushroom-based pharmaceuticals. Appl. Microbiol. Biotechnol. 2014, 98, 7661-7670. [CrossRef] [PubMed]

15. Gil-Ramírez, A.; Pavo-Caballero, C.; Baeza, E.; Baenas, N.; Garcia-Viguera, C.; Marín, F.R.; Soler-Rivas, C. Mushrooms do not contain flavonoids. J. Funct. Foods. 2016, 25, 1-13. [CrossRef]

16. Mattila, P.; Konko, K.; Eurola, M.; Pihlava, J.M.; Astola, J.; Vahteristo, L.; Hietaniemi, V.; Kumpulainen, J.; Valtonen, M.; Piironen, V. Contents of vitamins, mineral elements, and some phenolic compounds in cultivated mushrooms. J. Agric. Food Chem. 2001, 49, 2343-2348. [CrossRef]

17. Lo, K.M.; Cheung, P.C. Antioxidant activity of extracts from the fruiting bodies of Agrocybe aegerita var. alba. Food Chem. 2005, 89, 533-539. [CrossRef]

18. Kuo, H.C.; Lu, C.C.; Shen, C.H.; Tung, S.Y.; Hsieh, M.C.; Lee, K.C. Hericium erinaceus mycelium and its isolated erinacine A protection from MPTP-induced neurotoxicity through the ER stress, triggering an apoptosis cascade. J. Transl. Med. 2016, 14, 78. [CrossRef]

19. Li, I.; Lee, L.Y.; Tzeng, T.T.; Chen, W.P.; Chen, Y.P.; Shiao, Y.J.; Chen, C.C. Neurohealth properties of Hericium erinaceus mycelia enriched with erinacines. Behav. Neurol. 2018, 2018, 5802634. [CrossRef]

20. Bhatta, S.; Stevanovic Janezic, T.; Ratti, C. Freeze-drying of plant-based foods. Foods 2020, 9, 87. [CrossRef]

21. Association of Official Analytical Chemists (AOAC). Official Methods of Analysis, 16th ed.; Association of Official Analytical Chemists: Arlington, VA, USA, 1995.

22. Fernández-Ruiz, V.; Galiana, L.; Sánchez-Mata, M.C.; Chaya, C.; Roselló, S.; Cámara, M.; Torija, M.E.; Nuez, F. Internal quality characterization of fresh tomato fruits. HortScience 2004, 39, 339-345. [CrossRef]

23. Ruiz-Rodríguez, B.; Morales, P.; Fernández-Ruiz, V.; Sánchez-Mata, M.C.; Cámara, M.; Díez-Marqués, C.; Santayana, M.P.; Molina, M.; Tardío, J. Valorization of wild strawberry tree fruits (Arbutus unedo L.) through nutritional assessment and natural production data. Food Res. Int. 2011, 44, 1244-1253. [CrossRef]

24. Oyaizu, M. Studies on products of browning reactions: Antioxidative activities of products of browning reaction prepared from glucosamine. Jpn. J. Nutr. Diet. 1986, 44, 307-315. [CrossRef]

25. Delcour, J.A.; Varebeke, D.J.D. A new colourimetric assay for flavanoids in pilsner beers. J. Inst. Brew. 1985, 91, 37-40. [CrossRef] 
26. Velioglu, Y.S.; Mazza, G.; Gao, L.; Oomah, B.D. Antioxidant activity and total phenolics in selected fruits, vegetables, and grain products. J. Agric. Food Chem. 1998, 46, 4113-4117. [CrossRef]

27. Shimada, K.; Fujikawa, K.; Yahara, K.; Nakamura, T. Antioxidative properties of xanthan on the autoxidation of soybean oil in cyclodextrin emulsion. J. Agric. Food Chem. 1992, 40, 945-948. [CrossRef]

28. Ellman, G.L.; Courtney, K.D.; Andres, V., Jr.; Featherstone, R.M. A new and rapid colorimetric determination of acetylcholinesterase activity. Biochem. Pharmacol. 1961, 7, 88-95. [CrossRef]

29. Carradori, S.; Cairone, F.; Garzoli, S.; Fabrizi, G.; Iazzetti, A.; Giusti, A.M.; Menghini, L.; Uysal, S.; Ak, G.; Zengin, G.; et al. Phytocomplex characterization and biological evaluation of powdered fruits and leaves from Elaeagnus angustifolia. Molecules 2020, 25, 2021. [CrossRef]

30. Kawagishi, H.; Ando, M.; Sakamoto,H.; Yoshida, S.; Ojima, F.; Ishiguro, Y.; Ukai, N.; Furukawa, S. Hericenones $\mathrm{C}, \mathrm{D}$ and $\mathrm{E}$, stimulators of nerve growth factor (NGF)-synthesis, from the mushroom Hericium erinaceum. Tetrahedron Lett. 1991, 32, 4561-4564. [CrossRef]

31. Zhong, K.; Wang, Q. Optimization of ultrasonic extraction of polysaccharides from dried longan pulp using response surface methodology. Carbohydr. Polym. 2010, 80, 19-25. [CrossRef]

32. Yan, L.Y.; Yu, C.H.; Chen, J.; Li, X.X.; Wang, W.; Li, S.Q. Ultrasonic-assisted extraction optimized by response surface methodology, chemical composition and antioxidant activity of polysaccharides from Tremella mesenterica. Carbohydr. Polym. 2011, 83, 217-224. [CrossRef]

33. Choi, M.; Park, N.Y.; Woo, S.M.; Jeong, Y.J. Optimization of extraction conditions from Hericium erinaceus by response surface methodology. Korean J. Food Sci. Technol. 2003, 35, 777-782.

34. Yang, J.H.; Tseng, Y.H.; Lee, Y.L.; Mau, J.L. Antioxidant properties of methanolic extracts from monascal rice. LWT 2006, 39, 740-747. [CrossRef]

35. Puttaraju, G.N.; Venkateshaiah, S.U.; Dharmesh, S.M.; Urs, N.; Somasundaram, R. Antioxidant activity of indigenous edible mushrooms. J. Agric. Food Chem. 2006, 54, 9764-9772. [CrossRef] [PubMed]

36. Kalac, P. Chemical composition and nutritional value of European species of wild growing mushrooms. In Mushrooms: Types, Properties and Nutrition; Nova Science Publishers: Hauppauge, NY, USA, 2012; pp. 130-151.

37. Kalac, P. Chemical composition and nutritional value of European species of wild growing mushrooms: A review. Food Chem. 2009, 113, 9-16. [CrossRef]

38. Krzyczkowski, W.; Malinowska, E.; Herold, F. Erinacine A biosynthesis in submerged cultivation of Hericium erinaceum: Quantification and improved cultivation. Eng. Life Sci. 2010, 10, 446-457. [CrossRef]

39. Corana, F.; Cesaroni, V.; Mannucci, B.; Baiguera, R.M.; Picco, A.M.; Savino, E.; Ratto, D.; Perini, C.; Kawagishi, H.; Girometta, C.E.; et al. Array of metabolites in Italian Hericium erinaceus mycelium, primordium, and sporophore. Molecules 2019, 24, 3511. [CrossRef]

40. Friedman, M. Mushroom polysaccharides: Chemistry and antiobesity, antidiabetes, anticancer, and antibiotic properties in cells, rodents, and humans. Foods 2016, 5, 80. [CrossRef]

41. Charumathy, M.; Sudha, G.; Balakrishnan, P. Detection of antioxidant activity and bioactive constituents in the fruiting bodies of Hericium erinaceus pers-an edible mushroom. J. Pharm. Pham. Sci. 2016, 8, 152-156.

42. Rice-Evans, C.; Miller, N.; Paganga, G. Antioxidant properties of phenolic compounds. Trends Plant. Sci. 1997, 2, 152-159. [CrossRef]

43. Tsai, S.Z.; Tsai, H.L.; Mau, J.L. Antioxidant properties of Agaricus blazei, Agrocybe cylindracea, and Boletus edulis. LWT 2007, 40, 1392-1402. [CrossRef]

44. Kim, M.Y.; Seguin, P.; Ahn, J.K.; Kim, J.J.; Chun, S.C.; Kim, E.H.; Seo, S.H.; Kang, E.Y.; Kim, S.L.; Park, Y.J.; et al. Phenolic compound concentration and antioxidant activities of edible and medicinal mushrooms from Korea. J. Agric. Food Chem. 2008, 56, 7265-7270. [CrossRef] [PubMed]

45. Helano, S.A.; Barros, L.; Martins, A.; Queiroz, M.J.R.P.; Santos-Buelga, C.; Ferreira, I.C.F.R. Fruiting body, spores and in vitro produced mycelium of Ganoderma lucidum from Northest Portugal: A comparative study of the antioxidant potential of phenolic and polysaccharidic extracts. Food Res. Int. 2012, 46, 135-140. [CrossRef]

46. Li, H.; Park, S.; Moon, B.; Yoo, Y.B.; Lee, Y.W.; Lee, C. Targeted phenolic analysis in Hericium erinaceum and its antioxidant activities. Food Sci. Biotechnol. 2012, 21, 881-888. [CrossRef]

47. Seljelid, R. A water-soluble aminated $\beta 1-3 \mathrm{D}$-glucan derivative causes regression of solid tumors in mice. Biosci. Rep. 1986, 6, 845-851. [CrossRef] 
48. Nguyen, T.K.; Im, K.H.; Choi, J.; Shin, P.G.; Lee, T.S. Evaluation of antioxidant, anti-cholinesterase, and anti-inflammatory effects of culinary mushroom Pleurotus pulmonarius. Mycobiology 2016, 44, 291-301. [CrossRef]

49. Gursoy, N.; Sarikurkcu, C.; Cengiz, M.; Solak, M.H. Antioxidant activities, metal contents, total phenolics and flavonoids of seven Morchella species. Food Chem. Toxicol. 2009, 47, 2381-2388. [CrossRef]

50. Vamanu, E.; Nita, S. Antioxidant capacity and the correlation with major phenolic compounds, anthocyanin, and tocopherol content in various extracts from the wild edible Boletus edulis mushroom. BioMed Res. Int. 2012, 2013, 313-905.

51. Manjunathan, J.; Subbulakshmi, N.; Shanmugapriya, R.; Kaviyarasan, V. Proximate and mineral composition of four edible mushroom species from South India. Int. J. Biodivers. Conserv. 2011, 3, 386-388.

52. Sharif, S.; Mustafa, G.; Munir, H.; Weaver, C.M.; Jamil, Y.; Shahid, M. Proximate composition and micronutrient mineral profile of wild Ganoderma lucidum and four commercial exotic mushrooms by ICP-OES and LIBS. J. Food Nutr. Res. 2016, 4, 703-708.

53. Keong, C.Y.; Rashid, B.A.A.; Ing, Y.S.; Ismail, Z. Quantification and identification of polysaccharide contents in Hericium erinaceus. Nutr. Food Sci. 2007, 37, 260-271. [CrossRef]

54. Han, Z.H.; Ye, J.M.; Wang, G.F. Evaluation of in vivo antioxidant activity of Hericium erinaceus polysaccharides. Int. J. Biol. Macromol. 2013, 52, 66-71. [CrossRef]

Publisher's Note: MDPI stays neutral with regard to jurisdictional claims in published maps and institutional affiliations. 